\title{
Un retour des patronymes au Québec, 2005-2010 : au-delà des chiffres, des discours complexes entre égalité, identité et filiation
}

\section{A return to patronymics in Quebec, 2005-2010: behind the numbers - complex discourses between equality, identity and filiation}

\author{
Laurence Charton, Louis Duchesne, Denise Lemieux et Françoise-Romaine \\ Ouellette
}

Volume 44, numéro 1, printemps 2015

URI : https://id.erudit.org/iderudit/1032147ar

DOI : https://doi.org/10.7202/1032147ar

Aller au sommaire du numéro

Éditeur(s)

Association des démographes du Québec

ISSN

1705-1495 (numérique)

Découvrir la revue

Citer cet article

Charton, L., Duchesne, L., Lemieux, D. \& Ouellette, F.-R. (2015). Un retour des patronymes au Québec, 2005-2010 : au-delà des chiffres, des discours

complexes entre égalité, identité et filiation. Cahiers québécois de démographie, 44(1), 5-34. https://doi.org/10.7202/1032147ar
Résumé de l'article

Depuis 1981, le Code civil du Québec, dans la foulée de la reconnaissance du principe d'égalité des parents, permet d'attribuer à un enfant soit le nom de son père, soit le nom de sa mère, soit les deux noms réunis. La personne qui a reçu un nom composé peut le transmettre en totalité ou en partie à son enfant, combiné ou non avec le nom simple ou une partie du nom composé de l'autre parent (16 possibilités). Cet article propose une réflexion sur cette réforme à travers son impact sur les dynamiques de transmission du nom, sur la base des statistiques postérieures à la nouvelle législation (1980-2010) ainsi que de 25 entretiens autour de la naissance du premier enfant réalisés auprès de mères ou de pères d'enfants nés au cours de la période 2008-2013, incluant des parents porteurs d'un double nom. Cet article analyse, à partir des chiffres disponibles, une hausse de l'attribution du nom du père au cours des dernières décennies, succédant à une croissance du double nom dans les années 1980. Sans être généralisables, les entretiens suggèrent un accroissement de l'importance de la fonction connotative des discours entourant les processus de nomination, où s'entremêlent de façon complexe les enjeux de filiation, d'identité et d'égalité.
Tous droits réservés @ Association des démographes du Québec, 2015
Ce document est protégé par la loi sur le droit d'auteur. L’utilisation des services d'Érudit (y compris la reproduction) est assujettie à sa politique d'utilisation que vous pouvez consulter en ligne.

https://apropos.erudit.org/fr/usagers/politique-dutilisation/ 
Cahiers québécois de démographie

Vol. $44, \mathrm{n}^{\circ}$ 1, printemps 2015, p. 5-34

\title{
Un retour des patronymes au Québec, 2005-2010: au-delà des chiffres, des discours complexes entre égalité, identité et filiation
}

\author{
LAURENCE CHARTON*, LOUIS DUCHESNE ${ }^{* *}$, \\ DENISE LEMIEUX ${ }^{\star}$ ET FRANÇOISE-ROMAINE OUELLETTE*
}

Depuis 1981, le Code civil du Québec, dans la foulée de la reconnaissance du principe d'égalité des parents, permet d'attribuer à un enfant soit le nom de son père, soit le nom de sa mère, soit les deux noms réunis. La personne qui a reçu un nom composé peut le transmettre en totalité ou en partie à son enfant, combiné ou non avec le nom simple ou une partie du nom composé de l'autre parent (16 possibilités). Cet article propose une réflexion sur cette réforme à travers son impact sur les dynamiques de transmission du nom, sur la base des statistiques postérieures à la nouvelle législation (1980-2010) ainsi que de 25 entretiens autour de la naissance du premier enfant réalisés auprès de mères ou de pères d'enfants nés au cours de la période 2008-2013, incluant des parents porteurs d'un double nom. Cet article analyse, à partir des chiffres disponibles, une hausse de l'attribution du nom du père au cours des dernières décennies, succédant à une croissance du double nom dans les années 1980. Sans être généralisables, les entretiens suggèrent un accroissement de l'importance de la fonction connotative des discours entourant les processus de nomination, où s'entremêlent de façon complexe les enjeux de filiation, d'identité et d'égalité.

English abstract p. 34

\section{INTRODUCTION}

$\mathrm{L}$ es réformes législatives sur le nom observées au cours des quatre dernières décennies dans les pays occidentaux ont surtout affecté la nomination des personnes mariées et des enfants (Feschet, 2004; Lamesta et Baddeley, 2012 ; Louis-Pécha, 2010). Ces réformes ont contribué à favoriser une plus grande stabilité des noms des individus au cours de leur vie, par exemple en encadrant ou en limitant les changements de nom qui

* Centre Urbanisation, Culture, Société, Institut national de la recherche scientifique, Montréal (laurence.charton@ucs.inrs.ca)

** Institut de la statistique du Québec, Québec (retraité) 
seraient reliés au mariage. Elles ont aussi, sous d'autres angles, fait plus de place à la dimension subjective du nom, à la singularité de son porteur et à l'égalité des sexes, en autorisant par exemple qu'il ne soit plus transmis de génération en génération et en accordant plus de liberté de choix quant à la nomination des enfants (Fine et Ouellette, 2005).

Le nom constituerait ainsi plus généralement une question d'identité civile (en tant qu'élément primordial de l'état civil) et subjective (en tant que support à l'élaboration du sentiment de soi) ${ }^{1}$, de transmission entre générations et de négociation entre les parents (Charton et Lemieux, 2015). L'évolution des pratiques de nomination au Québec depuis la réforme du Code civil de 1980 en offre un exemple éloquent.

Plusieurs études en démographie historique, en anthropologie évolutive ou en génétique des populations basées sur les patronymes ont été effectuées au cours des dernières années (Brunet, Darlu et Barbero, 2008; Darlu et Degioanni, 2007; Darlu et collab., 2012; Lucchetti, Tasso, Amoruso et Caravello, 2011). Peu d'études démographiques ont porté par contre sur l'évolution du type de nom transmis au sein des générations (Chen et Cavalli-Sforza, 1983; Darlu, Degioanni et Ruffié, 1997; Duchesne, 2001). La question du choix du nom transmis à l'enfant est pourtant intéressante à étudier, notamment parce qu'elle permet de dégager d'importantes pistes de réflexion sur les rapports entre les partenaires au sein des couples et sur les liens intergénérationnels.

Cet article est divisé en trois parties. Dans un premier temps, nous présentons la réforme législative sur le nom au Québec et ses enjeux. Dans un deuxième temps, nous rappelons, à partir de données statistiques, l'évolution de l'attribution du nom de famille au Québec entre 1980 et 2005 et analysons la situation en 2010. Enfin, en nous basant sur un corpus d'entretiens réalisés en 2013 auprès de 25 parents québécois d'au moins un enfant de moins de 5 ans, nous explorons au moyen d'une analyse qualitative les processus entourant les choix de nomination.

\section{LA RÉFORME LÉGISLATIVE SUR LE NOM AU QUÉBEC ET SES ENJEUX}

Jusqu'en 1981, le nom qui était donné à l'enfant à sa naissance n'était régi au Québec par aucune loi, mais la coutume établie voulait que l'enfant légitime reçoive le nom de famille du père. Le fait pour un enfant de porter

1. Sur la dimension identitaire du nom et de l'état civil considérée à partir de l'exemple du changement de nom et de filiation des adoptés, voir Ouellette et Saint-Pierre (2011). 
plutôt le nom de sa mère pouvait d'ailleurs avoir un effet stigmatisant car il signifiait généralement que cet enfant était né hors mariage, n'avait pas été reconnu par son père et ne jouissait pas des mêmes droits que l'enfant légitime, c'est-à-dire celui né de parents mariés. Le nom patronymique ainsi transmis était désigné comme étant le «nom de famille», d'autant plus que la tradition voulait que les femmes prennent le nom de leur époux. Au Québec, l'inscription des actes de l'état civil sur un double registre par les ministres du culte depuis presque les débuts de la Nouvelle-France jusqu'en 1994 a probablement favorisé la stabilité des noms de famille et la très grande fréquence de certains de ces noms, dont les porteurs retracent la chaîne de leurs ascendants jusqu'au même ancêtre fondateur, particulièrement dans certaines régions de forte concentration patronymique (Bouchard, Desjardins-Ouellette, Markowski et Kouladjian, 1985; Desjardins, Bideau, Brunet, Charbonneau et Légaré, 2001; Duchesne, 2006; Garneau 1985). La reconnaissance par le droit québécois jusqu'en 1951 de l'autorité de l'usage du nom (qui peut ne pas reprendre le nom inscrit sur l'acte de naissance, voir Roch, 1949, p. 172; Deleury et Goubau, 1997, p. 226), associée à la décentralisation de l'état civil, a toutefois facilité aussi des changements informels de nom (Mellet, 2000, p. 14). Dans ce contexte, l'usage de surnoms a fait également apparaître de nouveaux patronymes, notamment quand il servait à distinguer entre elles des familles de même nom. Le surnom et le nom ont ainsi parfois formé ensemble un double nom ou ont été utilisés ensemble ou séparément de façon instable pendant quelques générations jusqu'à ce que l'un ou l'autre soit finalement adopté2. À la fin des années 1950, il semble cependant que la plupart des familles qui avaient acquis ainsi un nom double avaient renoncé à le transmettre (Roy, 1959, p. 38).

La réforme du droit de la famille entrée en vigueur le 2 avril 1981 (Gouvernement du Québec, 1980) a modifié cette donne. Le Code civil (CcQ) comporte depuis lors des règles précises d'attribution, d'utilisation et de changement du nom. Il prévoit notamment l'attribution à l'enfant, au choix de ses père et mère, d'un nom de famille simple ou composé d'au plus deux parties provenant des noms de famille de ses parents ${ }^{3}$, dans l'ordre choisi par eux (CcQ, art. 51). Ceci, sans imposer le choix du même nom pour les enfants d'une même fratrie. Suivant cette règle, la personne

2. "Sans ces surnoms, les mêmes noms de famille seraient presque deux fois plus nombreux qu'ils ne le sont à présent, ce qui n'est pas peu dire »(Roy, 1959, p. 138).

3. Si les parents portent le même nom, leur enfant peut théoriquement porter un nom double constitué par la répétition d'un même nom (exemple: Tremblay-Tremblay). 
qui a reçu un nom double peut le transmettre intégralement à son enfant, dans la mesure où l'autre parent accepte de ne pas donner son propre nom. Elle peut aussi ne transmettre qu'une partie de celui-ci, combinée ou non avec le nom simple ou une partie du nom double de l'autre parent. Pour l'enfant dont les deux parents ont un nom double, il existe ainsi jusqu'à 16 possibilités différentes de nomination.

Cette modification du système de nomination n'a constitué qu'un des nombreux aspects d'une réforme plus globale du droit familial qui visait, notamment, à consacrer juridiquement l'égalité entre les hommes et les femmes, l'égalité de tous les enfants indépendamment des circonstances de leur naissance et la liberté des personnes d'organiser leurs relations familiales. En rompant avec la coutume de transmettre à l'enfant le patronyme de son père ${ }^{4}$, elle mettait fin au marquage stigmatisant les enfants qui ne portent pas le nom de leur père. En permettant aux femmes de transmettre leur nom, elle s'inscrivait dans la foulée de la reconnaissance de l'autorité parentale partagée par les deux parents, qui avait été substituée au principe de la puissance paternelle en 1977. De plus, elle faisait écho à l'obligation faite aux femmes mariées de ne plus utiliser leur nom marital pour tous les actes de la vie publique. Plusieurs d'entre elles avaient déjà pris l'habitude au cours des années 1960 et 1970 de garder l'usage de leur nom de naissance et d'y adjoindre celui de leur mari, mais ces doubles noms féminins allaient quasiment disparaître du paysage onomastique québécois après 1981. Aujourd'hui, la majorité des noms doubles sont plutôt reçus à la naissance et portés par des individus des deux sexes tout au long de leur vie.

Cette nouvelle règle du droit civil n'accordant ni l'exclusivité, ni même la préséance, au nom du père a suscité beaucoup d'objections. Selon certains, elle allait créer une instabilité du nom d'une génération à l'autre et permettrait que des noms différents soient portés au sein d'une même famille, ce qui risquait d'affaiblir la cohésion familiale et compliquer la vie de tous les jours, par exemple la justification des liens parents-enfants. Elle réduirait le soutien symbolique que la transmission du nom du père apporte à la fonction paternelle, alors que les femmes bénéficient du fait que l'accouchement prouve sans aucun doute leur maternité. Enfin, les noms doubles seraient trop lourds à porter, parce que plus compliqués à prononcer et à écrire et souvent trop longs pour les formulaires standardisés.

L'aspect de la réforme qui a toutefois suscité les plus sérieuses résistances concerne la décision de laisser le libre choix du nom de l'enfant à ses

4. Ce paragraphe et les trois qui suivent reprennent sous une forme remaniée des extraits des pages 227 à 230 de Ouellette (2013). 
père et mère en n'imposant aucune règle de transmission automatique. En effet, au moment de nommer leurs propres enfants, les jeunes Québécois de la deuxième génération née après la réforme - surtout ceux dotés d'un nom double - auraient un jour à choisir eux-mêmes de transmettre intégralement le nom transmis par leurs parents, de le décomposer ou de l'abandonner. Les placer devant cette obligation de décider eux-mêmes de retransmettre ou pas l'héritage nominal de leurs parents (et grandsparents) s'écarte si radicalement de la norme traditionnelle d'inscription dans la parenté que certains y ont vu un ébranlement de l'ordre symbolique de la filiation, une menace à la structuration psychique des sujets humains ${ }^{5}$. Sans être aussi alarmiste, il était facile d'appréhender des conflits conjugaux, des traumatismes personnels ou des décisions irréfléchies chez ceux et celles qui auraient à assumer de tels choix. Certains groupes, notamment des groupes féministes, ont donc plutôt proposé la transmission automatique du nom de la mère aux filles et celle du nom du père aux garçons, ce qui aurait constitué des lignées paternelles et des lignées maternelles. D'autres ont préconisé la transmission systématique des deux noms en plaçant celui de la mère en premier pour les filles et celui du père en premier pour les garçons, de sorte qu'à la génération suivante chacun transmette uniquement la première partie de son nom à ses enfants. Là encore, les lignées maternelles et paternelles auraient été rendues visibles, tout autant que l'alliance parentale. Le Conseil du statut de la femme (CSF) a proposé l'attribution d'un nom double toujours composé de celui de la mère précédant celui du père; à la deuxième génération, la mère transmettrait la première partie de son nom et le père la deuxième partie du sien. En cas de rupture du couple parental, le CSF considérait que porter le nom de ses deux parents pourrait éviter à l'enfant séparé de l'un ou de l'autre des traumatismes inutiles (CSF, 1979, p. 12).

Malgré les critiques et les suggestions, les parlementaires ont privilégié le libre choix. Ce faisant, ils ont créé un système de nomination qui s'écarte d'une logique de transmission lignagère faisant passer un nom de génération en génération. Ce système permet aux parents d'attribuer à leur enfant un nom qui lui sera propre, qu'il pourrait ne partager avec personne et qu'il ne sera pas obligé de transmettre à son tour. Parce qu'il exige à chaque fois une négociation au sein du couple parental, il a déplacé dans la sphère privée l'enjeu politique de la reconnaissance de l'égalité des femmes face à la nomination des enfants (Cousteau Serdong, 2008).

5. Voir, par exemple, la position, nuancée et féministe, de Bergeron (1990). 
Aujourd'hui, 35 ans après la réforme du Code civil, quelle est l'importance du nom double dans la transmission du nom? Quel nom les pères et mères dotés par leurs parents d'un double nom (patronyme + matronyme, par exemple) ont-ils choisi de transmettre à leurs propres enfants? Observe-t-on des différences dans la transmission du nom à l'enfant selon qu'il s'agisse du père, de la mère ou des deux parents qui portent un nom double? C'est ce que nous allons étudier maintenant à travers l'analyse de l'évolution de l'attribution du nom au Québec depuis 1981. Ces analyses permettront de rendre compte non seulement de la part des noms doubles attribués pendant cette période, mais aussi de l'importance des fréquences de transmission matrilinéaire en regard des fréquences de transmission patrilinéaire. Nous accorderons par ailleurs une attention particulière à la structure (simple ou composée) des noms transmis en 2010 selon celle des noms des parents afin de rendre compte des éventuels effets intergénérationnels quand des porteurs de noms doubles arrivent à la parentalité.

\section{ÉVOLUTION DE L'ATTRIBUTION DU NOM DE FAMILLE AU QUÉBEC (1980-2010)}

Si, officiellement, ce n'est qu'à partir de 1981 que les parents québécois peuvent choisir le nom qu'ils souhaitent donner à leur enfant, certains parents n'avaient pas attendu le changement de la loi pour transmettre leurs deux noms respectifs à leur nouveau-né. Ainsi, en 1980, $2 \%$ des bébés avaient déjà reçu un nom double (nom de la mère suivi du nom du père ou inversement) (Duchesne, 2006). Près de $5 \%$ des nouveau-nés portaient par ailleurs exclusivement le nom de leur mère (le père étant inconnu dans la plupart de ces cas), tandis que $93 \%$ d'entre eux portaient exclusivement celui de leur père (tableau 1$)^{6}$.

6. La réforme entrée en vigueur au 2 avril 1981 comportait une disposition transitoire dont les parents de 9564 enfants déjà nés se seraient prévalus pour changer le nom de ceux-ci en nom composé (Deleury et Goubau 1997, p. 229) :

"Les père et mère d'un enfant mineur peuvent, dans les deux ans suivant la date d'entrée en vigueur de l'article 56.1 du Code civil du Bas-Canada, transmettre au ministre de la Justice une requête en vue d'attribuer à leur enfant mineur un nom composé d'au plus deux parties provenant des noms de ses père et mère. Cette requête est faite conformément aux dispositions de la Loi sur le changement de nom et d'autres qualités de l'état civil (L.R.Q., c. C-10). Cependant, les requérants sont dispensés de donner les avis prévus par les articles 5 et 9 de ladite loi. » (Gouvernement du Québec, 1980, art. 78) 
TABLEAU 1 Attribution (\%) du nom aux enfants, Québec, 1980, 1992, 2005 et 2010

\begin{tabular}{|l|c|c|c|c|}
\hline Nom transmis à l'enfant & $\mathbf{1 9 8 0}$ & $\mathbf{1 9 9 2}$ & $\mathbf{2 0 0 5}$ & $\mathbf{2 0 1 0}^{\mathbf{a}}$ \\
\hline Père & 92,5 & 71,2 & 82,2 & 84,9 \\
\hline Mère & 4,9 & 6,3 & 4,7 & 4,3 \\
\hline Père-Mère $^{b}$ & 0,3 & 4,5 & 4,2 & 4,9 \\
\hline Mère-Père $^{c}$ & 1,5 & 17,2 & 7,8 & 4,9 \\
\hline Père=Mèred $^{\text {d }}$ & 0,8 & 0,8 & 1,1 & 0,9 \\
\hline Autre & - & - & - & 0,1 \\
\hline Total & 100,0 & 100,0 & 100,0 & 100,0 \\
\hline
\end{tabular}

a La méthodologie utilisée en 2010 n'est pas exactement la même que les années précédentes

${ }^{b}$ Nom du père suivi du nom de la mère.

${ }^{c}$ Nom de la mère suivi du nom du père.

${ }^{\mathrm{d}}$ Les parents ont le même nom.

Remarque: il peut s'agir d'une partie d'un nom si un parent a un nom composé.

Sources: pour les années 1980, 1992 et 2005: ISQ, 2006, p.123; pour 2010, exploitation du Registre des événements démographiques du Centre d'accès aux données de recherche de l'ISQ (CADRISQ).

L'importance du nom double a évolué ensuite rapidement, atteignant un sommet à $22 \%$ en 1992 avant de connaitre une forte diminution dans les décennies suivantes, passant de $12 \%$ en 2005 à $10 \%$ en 2010. Cette diminution de la proportion des noms de famille doubles au cours des deux dernières décennies est sans doute liée, au moins en partie, à une augmentation des enfants nés de parents immigrants qui conservent peutêtre davantage la coutume d'attribuer seulement le nom du père à leurs enfants. Depuis 1990 en effet, la proportion des naissances provenant de parents nés à l'étranger a fortement augmenté : selon les données de l'Institut de la Statistique du Québec (ISQ, 2014), $17 \%$ des enfants nés au Québec en 2010 avaient deux parents nés à l'extérieur du Canada, comparativement à seulement $8 \%$ en 1990 . Notons par ailleurs que si la proportion de noms doubles peut être estimée facilement jusqu'au milieu des années 2000 en additionnant les noms Père-Mère et Mère-Père, les données de 2010 présentées dans le tableau 1 ne permettent pas par contre de percevoir toute l'évolution de la réalité onomastique du nom double au Québec. En effet, l'enfant qui reçoit en 2010 le nom double de son père dans son intégralité est comptabilisé parmi ceux recevant uniquement le nom du père.

La hausse des noms doubles observée dans les années qui ont suivi le changement de la loi pouvait laisser cependant penser que le Québec s'orientait progressivement vers un système égalitaire dans la transmission 
du nom, voire même vers un système matrilinéaire (Duchesne, 2001). Ainsi, en 1992, le nom double le plus souvent transmis était composé du nom de la mère suivi de celui du père $(17 \%$ contre $5 \%)$. Si la primauté du nom de la mère dans les noms doubles a prévalu jusqu'en 2005 (8 \% contre $4 \%$ ), ce n'est plus le cas en 2010, la moitié des noms doubles étant composée par celui de la mère suivi de celui du père $(5 \%)$ et l'autre moitié de celui du père suivi de celui de la mère $(5 \%)$. Peu de différences s'observent par ailleurs dans le choix du nom transmis à l'enfant selon le sexe de celui-ci. On relève à peine une légère préférence pour le nom de la mère chez les filles (nom de la mère uniquement + nom double Mère-Père : $9,4 \%$ contre $8,9 \%$ chez les garçons) (tableau 2).

TABLEAU 2 Attribution (\%) du nom aux enfants en 2010 selon le sexe de l'enfant, Québec

\begin{tabular}{|l|c|c|}
\hline Nom transmis selon le sexe de l'enfant & Garçons & Filles \\
\hline Père & 85,1 & 84,7 \\
\hline Mère & 4,1 & 4,5 \\
\hline Père-Mère $^{\mathrm{a}}$ & 5,0 & 4,9 \\
\hline Mère-Père $^{\mathrm{b}}$ & 4,8 & 4,9 \\
\hline Père=Mère $^{\mathrm{c}}$ & 0,9 & 0,9 \\
\hline Autre & 0,1 & 0,1 \\
\hline Total & 100,0 & 100,0 \\
\hline
\end{tabular}

a Nom du père suivi du nom de la mère.

${ }^{b}$ Nom de la mère suivi du nom du père.

c Les parents ont le même nom.

Remarque: il peut s'agir d'une partie d'un nom si un parent a un nom composé.

Source: exploitation du Registre des événements démographiques du Centre d'accès aux données de recherche de I'ISQ (CADRISQ).

Depuis 2005, le nom composé du nom des deux parents diminue ainsi en faveur du nom du père exclusivement, une tendance qui avait déjà été observée sur le terrain (LeGall et Meintel, 2005; Lemieux, 2005) : le nom du père transmis aux enfants est passé de $71 \%$ en 1992 à $82 \%$ en 2005, pour atteindre $85 \%$ en 2010 (tableau 1). Comment expliquer cette baisse du nom double chez les nouveau-nés? Quel est le profil de nomination des parents transmettant aujourd'hui un nom double? Le nom porté par les parents joue-t-il en d'autres termes un rôle sur le choix du nom transmis à l'enfant? 
Pour apporter des éléments d'explications à ces questions, notamment pour déterminer le type de nom transmis aux enfants compte tenu du nom des parents nous avons eu recours au fichier des bulletins de naissances vivantes au Québec compilés par l'ISQ pour la dernière année disponible à la date de notre demande, soit $2010^{7}$. Toutes les analyses ont été réalisées au Centre d'accès aux données de recherche de l'ISQ (CADRISQ). Pour des raisons liées au coût d'accès aux données, le fichier des naissances ne comprenait que le nom de l'enfant et les noms de ses père et mère. Pour les enfants issus de couples lesbiens, seul le nom d'une mère a été enregistré dans le fichier statistique des naissances. Lorsque le nom de l'enfant ne correspondait ni au nom de la mère ni à celui du père, le choix de nom a été consigné dans la modalité " autre » (cas rares : 0,1\% en 2010). Enfin, si le nombre des naissances comptabilisé en 2010 au Québec est de 88436, les analyses réalisées dans le cadre de cet article n'ont porté que sur 79120 naissances, en raison de la non-prise en compte des naissances de l'Outaouais et de celles dont le nom de l'enfant n'est pas déclaré (voir encadré 1).

L'âge moyen à la naissance des enfants est, au Québec en 2010, d'environ 30 ans pour les femmes et 33 ans pour les hommes (ISQ, 2014). La plupart des parents d'enfants nés cette année-là appartiennent donc aux générations du début des années 1980, soit celles du début de la vague des noms doubles. Ces parents sont ainsi assez peu nombreux à porter un nom double : environ $5 \%$ des mères et des pères (tableau 3 ).

Pour rendre compte de l'importance du nom double dans la transmission du nom (transmission du nom de chaque parent ou d'un nom double de l'un des parents), nous allons maintenant traiter la question des noms doubles de façon indépendante de celle du choix du nom transmis par chaque parent et présenté plus haut.

En 2010, si 9,8 \% des nouveau-nés ont reçu un nom de chacun de leurs parents, $11,4 \%$ des enfants ont hérité d'un nom double. Ainsi, 1,6 \% des noms doubles transmis en 2010 ont été hérités de l'un des parents. Il n'y a pas de différences observées dans la proportion du nom double transmis selon que l'enfant soit un garçon ou une fille (tableau 3).

7. Nous remercions Fabienne Cléophat-Jolicoeur, agente-conseil aux services d'accès aux données à des fins de recherche à la Direction des services informationnels et technologiques de l'Institut de la statistique du Québec pour son aide dans la préparation des demandes d'accès aux données. 


\section{Encadré 1: Processus d'enregistrement des naissances vivantes au Québec}

Il existe au Québec un double enregistrement des naissances : un enregistrement au registre de l'état civil du Québec tenu par le directeur de l'état civil et un enregistrement sociosanitaire au registre des événements démographiques géré par la Direction des statistiques sociodémographiques de l'Institut de la Statistique du Québec (ISQ). Depuis 1994 (articles 103 et suivants du Code civil du Québec), le directeur de l'état civil est le seul officier de l'état civil (auparavant les ministres du culte ainsi que les secrétaires ou greffiers des municipalités étaient les officiers d'état civil et tenaient les registres des naissances). L'ISQ fournit aux établissements de naissance les exemplaires vierges à remplir des constats de naissance dressés par les accoucheurs et des déclarations de naissance des parents, qui sont envoyés directement au directeur de l'état civil, ainsi que ceux du bulletin de naissance vivante ( $S P-1$ ), qui est destiné au registre des événements démographiques de l'ISQ. Le cheminement de ce bulletin est rapide car il est envoyé à l'ISQ directement par l'établissement de naissance. Les parents ont par contre 30 jours pour faire parvenir la déclaration de naissance au directeur de l'état civil. Les données statistiques présentées dans cet article proviennent exclusivement des données enregistrées à l'ISQ à partir des bulletins de naissance vivante. Ce sont toutefois les nom et prénoms figurant sur la déclaration de naissance qui sont inscrits à l'acte de naissance de l'enfant et ils peuvent différer de ceux inscrits sur le bulletin de naissance vivante car certains parents modifient leur choix entre les deux déclarations. Les bulletins de naissance comportent par ailleurs des données manquantes, en particulier l'absence de déclaration du nom de l'enfant dans un peu moins de $1 \%$ des naissances. Les données manquantes ont été réparties au prorata des données connues.

Lorsqu'on regarde maintenant le type de nom (simple ou double) des parents ayant transmis à leur enfant un nom double en $2010(11,4 \%$ des enfants), on constate que ce sont les parents qui ont chacun un nom double qui donnent le plus souvent $(74,6 \%)$ un nom double à leur enfant (tableau 4). Un quart d'entre eux abandonne néanmoins le double nom au profit d'un nom simple. Les parents qui portent un nom simple sont pour 
TABLEAU 3 Répartition (\%) des noms simples et doubles chez les parents et les enfants nés en 2010, Québec

\begin{tabular}{|l|c|c|c|}
\hline \multirow{2}{*}{$\begin{array}{l}\text { Père, mère et } \\
\text { enfants }\end{array}$} & \multicolumn{3}{|c|}{ Type de nom (\%) } \\
\cline { 2 - 4 } & Simple & Double & Total \\
\hline Père $^{\mathrm{a}}$ & 94,7 & 5,3 & 100,0 \\
\hline Mère & 95,5 & 4,5 & 100,0 \\
\hline Enfants & 88,6 & 11,4 & 100,0 \\
\hline \multicolumn{1}{|c|}{ Garçons } & 88,6 & 11,4 & 100,0 \\
\hline \multicolumn{1}{|c|}{ Filles } & 88,5 & 11,5 & 100,0 \\
\hline
\end{tabular}

${ }^{a}$ Les non déclarés sont répartis au prorata.

Source: exploitation du Registre des événements démographiques du Centre d'accès aux données de recherche de I'ISQ (CADRISQ).

leur part les plus réfractaires aux noms doubles et choisissent plus de neuf fois sur dix $(92,5 \%)$ un nom simple pour leur enfant. Enfin, quand il y a un seul parent qui porte un double nom, un enfant sur trois $(33 \%)$ reçoit quant à lui un nom double. Il existe néanmoins un écart dans le type de nom transmis (simple ou double) selon le porteur du nom double (mère ou père) chez les parents porteurs chacun d'un type de nom différent (double et simple). Les pères qui portent un nom double perpétuent en effet plus fréquemment la transmission du double nom à leur enfant que les mères. Ainsi, lorsque le père porte un nom simple et la mère un nom double, $74,4 \%$ des enfants héritent d'un nom simple. Dans le cas contraire (père nom double et mère nom simple), seul un peu plus de la moitié des enfants porte un nom simple $(51,2 \%)$.

Si ces résultats montrent un certain abandon du nom double dans la génération 2010 - puisque $62,3 \%$ des enfants de parents porteurs d'au moins un nom double ne portent qu'un nom simple - ils soulignent aussi l'écart existant selon qui (mère ou père) porte le double nom. Il semble ainsi que la transmission de "l'héritage » du nom soit plus difficile pour les femmes ayant porté un double nom que pour les hommes, notamment lorsque le père de leur enfant ne porte qu'un nom simple. 
TABLEAU 4 Répartition (\%) des noms simples et doubles des enfants selon le type de nom des parents, Québec, 2010

\begin{tabular}{|l|c|c|c|c|}
\hline \multirow{2}{*}{ Type de nom des parents $^{\mathbf{a}}$} & \multicolumn{2}{|c|}{ Type de nom des enfants (\%) } & \multirow{2}{*}{$\begin{array}{c}\text { Effectif } \\
\text { non } \\
\text { pondéré }\end{array}$} \\
\cline { 2 - 4 } & Simple & Double & Total & 68849 \\
\hline 2 parents nom simple & 92,5 & 7,5 & 100,0 & 1175 \\
\hline 2 parents nom double & 25,4 & 74,6 & 100,0 & 9 098 \\
\hline 1 parent nom double, l'autre simple & 67,0 & 33,0 & 100,0 & 2891 \\
\hline Père nom double, mère nom simple & 51,2 & 48,8 & 100,0 & 5844 \\
\hline Père nom simple, mère nom double & 74,4 & 25,6 & 100,0 & 10273 \\
\hline 1 ou 2 parents nom double & 62,3 & 37,7 & 100,0 & 192 \\
\hline Ensemble & 88,6 & 11,4 & 100,0 & 79122 \\
\hline
\end{tabular}

${ }^{a}$ Comprend les pères non déclarés.

Source: Exploitation du Registre des événements démographiques du Centre d'accès aux données de recherche de l'ISQ (CADRISQ).

TABLEAU 5 Noms attribués aux enfants de 2 parents porteurs d'un nom simple ou d'un parent porteur d'un nom double, Québec, 2010 (effectifs et proportions)

\begin{tabular}{|l|c|c|c|c|}
\hline $\begin{array}{l}\text { Nom des } \\
\text { enfants }\end{array}$ & $\begin{array}{c}\text { 2 parents avec } \\
\text { nom simple }\end{array}$ & $\begin{array}{c}\text { Effectif non } \\
\text { pondéré }\end{array}$ & $\begin{array}{c}\text { 1 parent avec } \\
\text { nom double }\end{array}$ & $\begin{array}{c}\text { Effectif non } \\
\text { pondéré }\end{array}$ \\
\hline Père & 87,5 & 60218 & 71,5 & 6507 \\
\hline Mère & 4,1 & 2795 & 6,3 & 570 \\
\hline Père-Mère & 3,1 & 2117 & 12,9 & 1177 \\
\hline Mère-Père & 4,3 & 2933 & 9,1 & 826 \\
\hline Père=Mère & 1,0 & 711 & - & - \\
\hline Autre & 0,1 & 75 & 0,2 & 15 \\
\hline Total & 100,0 & 68849 & 100,0 & 9095 \\
\hline
\end{tabular}

Source: Exploitation du Registre des événements démographiques du Centre d'accès aux données de recherche de l'ISQ (CADRISQ).

En croisant les noms simples et doubles de chaque parent selon le type de nom transmis aux enfants, on constate que lorsque les deux parents ont un nom simple, $87,5 \%$ des enfants portent le nom du père (pour $4,1 \%$ celui de la mère) (tableau 5). Parmi les $7,4 \%(3,1+4,3)$ de ceux qui optent pour un nom double, $58,1 \%(4,3 / 7,4)$ choisissent le nom Mère-Père. 
Chez les couples dont l'un des parents porte un nom double, $71,5 \%$ d'entre eux transmettent uniquement le nom du père à leur enfant (pour $6,3 \%$ celui de la mère) (tableau 5). Ceux qui ont reçu un nom de chacun des parents (22\%) ont par ailleurs majoritairement hérité $(58,6 \%$, soit 12,9/22) d'un nom Père-Mère.

Enfin, concernant les parents qui ont chacun un nom double, la majorité d'entre eux transmettent un nom provenant des deux parents à leurs enfants $(60 \%$, soit $50,7+9,3)$, et le plus souvent, $84,5 \%(50,7 / 60)$, un nom tiré d'une partie du nom du père et d'une partie du nom de la mère (tableau 6). Près de deux enfants sur cinq ( $38 \%)$ n'héritent cependant que $\mathrm{du}$ nom du père (une partie ou son double nom). Cette transmission comprend dans $64,2 \%$ des cas un nom simple et dans $35,8 \%$ le nom double du père (tableau 6). Il est rare par contre que la mère transmette seule son nom $(1,4 \%$ des cas et pour la plupart il ne s'agit que d'une partie du nom de la mère). Ces observations nuancent ainsi quelque peu les discours voulant que la diminution des noms doubles chez les nouveaunés soit le fait que les parents porteurs de ce type de nom l'apprécient peu. Elles soulignent aussi la place renforcée du nom du père dans la transmission du nom aux enfants.

TABLEAU 6 Noms attribués aux enfants de 2 parents porteurs d'un nom double, Québec, 2010 (effectifs et proportions)

\begin{tabular}{|l|c|c|}
\hline Nom des enfants & 2 parents avec nom double & Effectif non pondéré \\
\hline Père & 38,0 & 446 \\
\hline nom simple & 24,4 & 286 \\
\hline nom double & 13,6 & 160 \\
\hline Mère & 1,4 & 17 \\
\hline Père-Mère & 50,7 & 596 \\
\hline Mère-Père & 9,3 & 109 \\
\hline Père=Mère & - & - \\
\hline Autre & 0,6 & 7 \\
\hline Total & 100,0 & 1175 \\
\hline
\end{tabular}

Source: Exploitation du Registre des événements démographiques du Centre d'accès aux données de recherche de l'ISQ (CADRISQ).

En définitive, si l'essor des noms doubles composés des noms de chaque parent dans les années 1980 avait permis une visibilité plus grande 
du nom des mères, notamment à travers la vague des noms doubles «matrilinéaires» (nom de la mère suivi du père), il semble qu'avec la diminution des noms doubles composés de celui des père et mère (à la faveur des noms simples du père ou du nom double du père), cela ne soit plus le cas que d'un petit groupe d'enfants des générations de la fin du $\mathrm{xx}^{\mathrm{e}}$ et du début du xxi ${ }^{\mathrm{e}}$ siècle.

\section{MOTIVATIONS ET REPRÉSENTATIONS ENTOURANT LES CHOIX DE NOMINATION : RÉCITS DE PARENTS}

Pour comprendre les motivations associées au choix du nom transmis aux enfants, nous avons entrepris, parallèlement aux analyses quantitatives, une étude qualitative portant sur la nomination d'un premier enfant dans les familles québécoises entre 2008 et 2013. Nous souhaitions explorer plus précisément les effets de la réforme du Code civil sur la deuxième génération des enfants nés après son entrée en vigueur. Des entretiens semidirigés ont été réalisés en 2013 à Montréal et en région, auprès de 15 mères et 10 pères francophones (dont trois couples mixtes: mère québécoise et père d'Amérique du Sud, d'Asie ou d'Afrique), parents d'au moins un enfant de moins de 5 ans $^{8}$. Tous les parents rencontrés ont eu leur enfant dans le cadre d'un couple hétérosexuel et vivaient avec l'autre parent à l'annonce de la grossesse mais, à la date de l'enquête, trois d'entre eux (deux femmes et un homme) sont séparés. Huit parents sont mariés ou planifient de se marier prochainement, alors que les 17 autres vivaient en union de fait. Les parents rencontrés appartiennent à des profils sociodémographiques différents selon le niveau d'études, le type d'activité professionnelle, le lieu de résidence et le revenu du ménage. Les premiers parents ont été recrutés par voie d'annonces diffusées auprès d'organismes œuvrant auprès de jeunes familles (centres de la petite enfance, garderies...) et par l'intermédiaire des réseaux personnels de l'équipe de recherche. La méthode boule-de-neige a ensuite été retenue.

Les profils de nomination représentés dans cet échantillon sont tout autant variés, bien que reflétant la persistance d'un mode de nomination patronymique. Ainsi, à la naissance de leurs enfants, quinze parents sont dans un couple dont les deux conjoints portent le nom de leur père, neuf parents dans un couple où l'un des conjoints porte un nom composé et l'autre un nom simple, celui de son père, et une mère porte un patronyme

8. Les entretiens ont été réalisés par Mélanie Pearson, étudiante à la maîtrise en sociologie à l'Université de Montréal. 
et son conjoint un matronyme. Le nom transmis aux enfants a été dans dix-sept cas le nom du père seulement, dans cinq cas un nom composé (Père-Mère ou Mère-Père), et dans trois cas un nom par alternance (nom du père ou de la mère au premier enfant et nom de la mère ou du père au deuxième). Nous n'avons pas dans cet échantillon d'enfant ayant hérité exclusivement du nom de sa mère ou du nom double de l'un de ses parents. Le profil de notre échantillon reste toutefois assez proche de la répartition des noms transmis aux enfants en 2010 selon le type de nomination de leurs parents observée dans la deuxième partie de cet article. Ainsi, sur quinze couples de parents porteurs d'un nom patronymique simple, douze donnent à leurs enfants le patronyme du père et, sur neuf couples de parents dont l'un d'entre eux porte un nom double, quatre donnent un nom simple (celui du père) à leurs enfants.

Pour mieux cerner les raisons associées au choix d'un nom simple ou double, nous avons regroupé dans un premier temps les témoignages de parents qui ont choisi de transmettre un nom simple, celui du père, à leur premier enfant (soit 17 sur les 25). Ces parents ont par ailleurs donné ou prévu de donner le même nom à l'ensemble de la fratrie. Nous verrons que les discours et les parcours qui aboutissent à une telle décision sont très différenciés et dépendent en partie du nom que portent les parents et de leur vécu en regard de ce nom. Nous examinerons ensuite les parcours de ceux qui ont choisi d'attribuer un nom double à leur enfant. Enfin, nous porterons notre attention sur le choix d'une attribution alternée des noms du père et de la mère aux enfants successifs du couple. Tous les noms et prénoms présentés dans cet article sont des pseudonymes.

\section{Transmettre le nom du père}

\section{La transmission patronymique: une règle coutumière}

Plusieurs parents vont spontanément évoquer la tradition, de manière plus ou moins directe, pour justifier leur choix d'avoir transmis un patronyme à leur enfant. Certains disent aussi qu'ils n'en ont même pas discuté, que ce choix s'est fait "naturellement ", que c'est comme ça que ça s'est toujours fait. C'est le cas par exemple de David Blais, dont le témoignage indique d'ailleurs une méconnaissance des règles de nomination quand l'un des parents a un nom double. Il dit:

Moi je voulais donner le nom à mon gars, puis ma blonde voulait donner mon nom de famille à mon gars. Tu sais, c'était pour suivre la tradition. Normalement, l'enfant a plus le nom du père 
que de la mère. Puis on voyait pas le besoin non plus d'intégrer les noms de famille de ma blonde. Déjà que, elle a deux noms de famille; on (ne) voulait pas qu'il ait trois noms de famille, puis des affaires de même. (David Blais, 26 ans, conjoint de fait, électricien, un fils, vit en région)

Une parenté définie comme tissée serrée apparaît en toile de fond du choix de Johanne Turmel. Elle explique :

Pour moi, c'était naturel qu'il ait le nom du père. Mon frère va peut-être avoir des garçons un jour, ça va pouvoir continuer la lignée. Puis sinon, bien, mes cousins auront des gars. Rire] Je voyais pas l'intérêt, puis vu que Martial est enfant unique, je pense que ses parents trouvaient ça important aussi qu'il ait son nom. (Johanne Turmel, 28 ans, conjointe de fait, enseignante, un fils, vit en région)

Invitée à préciser sa pensée, elle considère que les deux noms peuvent être donnés lorsqu'un "bébé-accident » survient dans un couple qui n'est pas solide ou envisage de se séparer. Elle cite ainsi l'exemple de ses cousins qui ont transmis un double nom à leur enfant et qui se sont séparés depuis.

Pour Victoria Falardeau, la transmission du patronyme à ses enfants est aussi vécue comme une évidence, à la fois parce que c'est la coutume et par rejet du double nom. Elle s'explique :

Je pense que ça a toujours été convenu que ça serait son nom de famille à lui, que le père donnait son nom de famille à l'enfant. Bien, à la base on (ne) voulait pas un prénom composé parce qu'étant donné que je suis enseignante, je trouve ça long, les enfants qui ont à inscrire leur prénom composé. Ça fait que là je me disais, on n'ira pas choisir en plus un nom de famille composé étant donné que c'est déjà long, écrire un prénom composé. Ça fait que là on élimine le prénom composé, on élimine le nom de famille. (Victoria Falardeau, 25 ans, mariée, enseignante, une fille, vit en région)

Lucie Desmarais aurait aimé pour sa part transmettre son nom, mais sans que ses filles aient à porter un double nom. Elle se serait, dit-elle, contentée d'une initiale :

Bien, c'est ce qu'ils m'ont dit, à l'état civil : je [ne] pouvais pas lui donner les deux noms, puis qu'elle en porte juste un. Ça devenait vraiment compliqué parce que toutes ses cartes d'assurance, 
toutes ses inscriptions à l'école, il aurait fallu que tout ça soit aux deux noms, bien, à nos deux noms. [...] Je trouvais ça beau, Margaret D.-Rondeau, mais je voulais pas l'appeler Margaret-D. (Lucie Desmarais, 28 ans, mariée, secrétaire, deux filles, vit en région)

Son témoignage reprend aussi toute la panoplie des arguments pour appuyer le choix du nom du père en référence à sa propre expérience et aux normes de son milieu :

Moi, je porte juste le nom de mon père, puis je suis pas morte. [...] Tu sais, ça ne perpétuerait pas le nom des Desmarais parce qu'elle, si elle a des enfants, elle leur donnera pas le nom Desmarais. Ses enfants vont s'appeler probablement par le nom de leur père ou... [...] Ça me donne rien de lui donner mon nom de famille parce que c'est mon frère qui peut perpétuer le nom des Desmarais. Moi je peux pas, là. À moins d'être monoparentale ou je ne sais pas quoi, là, d'avoir un enfant pas de père, qui s'appellerait Desmarais.

Et puis, continue-t-elle :

Ça fait prétentieux, puis je dis tout le temps: tu aimes pas ton enfant pour lui donner autant de noms que ça. [...] Ça fait péteux de broue. Ça fait hautain. [...] égoïste, ça fait : moi je veux que mon enfant porte le nom.

Pour Lucie Desmarais, le patronyme transmis à ses filles signe en quelque sorte la stabilité de son couple et de la famille. Elle ajoute :

On est tellement habitué que ce soit le nom du père, le père qui est le chef de la famille, patati patata, qu'automatiquement c'est sûr que si je vois un enfant qui porte le nom de sa mère, je vais me dire : ça marche pas, les deux. Pour moi ça reflète un problème conjugal.

La référence à la tradition de transmettre le patronyme aux enfants est encore plus explicite dans les entrevues avec des personnes d'origines étrangères (à l'exception d'un père latino-américain marié à une Québécoise) ou de couples mixtes, qui disent que les noms doubles ça n'existe pas dans leur culture ou dans la culture de leur conjoint, comme Barthélémy Bossé (30 ans, marié, jardinier, une fille, vit en région), d'origine africaine et Nathalie Bélanger ( 32 ans, mariée, employée administrative, une fille, vit à Montréal), épouse d'un immigrant bhoutanais. 


\section{Le patronyme, marqueur de filiation et de paternité}

L'obligation de choisir qu'imposent les règles de l'état civil suscite une autre forme de discours chez des couples qui se disent par ailleurs non traditionalistes. Ils appuient leur décision de s'en tenir à la transmission du patronyme principalement sur une réflexion concernant les rôles maternels et paternels.

Pierre-Carl Sauvé, en évoquant les discussions à ce sujet dans son couple, exprime avec humour une certaine résistance aux nouvelles possibilités offertes. Il dit :

C’est sûr qu'on en a parlé, mais sûrement moins que du prénom. Pour moi, par définition, ça allait être le nom de famille du père, mais j'ai l'impression que c'est répandu. Je connais pas beaucoup d'enfants qui ont le nom de la mère. À moins d'avoir [...] des parents qui ont un métier, mettons... policiers, puis on veut pas qu'il ait le même nom que le père, ou... (Pierre-Carl Sauvé, 26 ans, conjoint de fait, journalier, une fille, vit en région).

Il se moque un peu de l'idée que son nom le rattacherait à une lignée à faire valoir et il souligne plutôt l'identité particulière que lui confère son nom de famille pas très courant :

Quand je me présente, je [ne] me présente pas comme fils de Pierre Sauvé, petit-fils de, tu sais, la grande lignée, là, de Louis XIV à Sauvé. Mais oui, oui, je suis fier de mon nom parce qu'il [n'] y en a pas beaucoup, puis en même temps, bien, c'est à moi, c'est mon identité. Je veux dire, c'est à moi d'en faire un petit peu aussi [pour] qu'est-ce que j'ai envie que les gens perçoivent de mon nom de famille.

Il prend par ailleurs à partie le vocabulaire utilisé pour présenter les parents dans les formulaires de déclaration de naissance dénonçant l'absence de référence explicite au père :

Non, il [n'] y a même pas «paternel », je pense. Dans les documents, la mère est très importante. Le père, ils font même pas mention du père. C'est, genre, "l'autre parent ». Le père est comme pas important [...], on dirait. Oui, je sais qu'il est très important le père, mais pour le directeur de l'état civil, le père...

Le fait que sa fille porte son nom suscite chez lui émotion et fierté :

Ça, c'est une petite partie de moi. [...], c'est sûrement la plus belle chose que j'ai pu faire de ma vie, c'est-à-dire donner la vie. Donc 
oui, je pense que le fait qu'elle ait mon nom de famille, chaque fois que quelqu'un va me le dire, c'est comme une belle reconnaissance. Oui, le nom de famille, bien, tu sais, c'est une appartenance, là.

Confronté à l'idée que la mère aussi pourrait aimer transmettre son identité, il commente :

Je pense que c'est un peu dommage pour elle, mais en même temps je pense que c'est un choix qui s'effectue à deux, puis je crois que c'est d'ailleurs pour ça qu'il y a des conflits parfois dans le couple, peut-être pas le nôtre... Mais je pense que c'est pour ça qu'il y a des gens qui portent les deux noms de famille, parce que peut-être que ça a été un trop gros dilemme, ou je pense qu'ils voulaient porter les deux tout simplement, avoir toute l'appartenance dans leur nom. Je [ne] sais pas trop comment l'exprimer, mais ce serait bien que la mère soit aussi dans le nom, c'est sûr. En fait, ça m'aurait pas tant dérangé que ce soit le nom de la mère.

Cette ouverture relative à la transmission du nom de l'autre parent est aussi présente chez Félix Lacroix qui, de façon informelle, s'est mis luimême à porter le nom de sa mère dans sa vie professionnelle. Pour leur enfant, par méconnaissance des règles de transmission et pour ne pas déroger à la tradition familiale, le couple a décidé toutefois de ne pas lui transmettre leurs deux noms. Il raconte :

Rapidement, on était venus à l'idée que, non, on donnerait pas deux noms de famille. On était d'accord dès le départ là-dessus. [...] Honnêtement ça m'aurait pas dérangé. Tu sais, c'est pas une question de lignée ou que ce soit mon nom, puis tout ça. On a décidé d'en donner juste un. Ça, on était d'accord que deux noms, (à) un moment donné ça se dédouble, puis ses enfants vont en avoir quatre, tout ça. On a décidé d'en donner un. (Félix Lacroix, 35 ans, conjoint de fait, informaticien, une fille, Montréal)

Généalogiste amateur, Félix Lacroix souligne également son attachement à sa famille en trouvant le prénom de sa fille dans sa lignée paternelle et en cherchant celui de son prochain enfant dans la lignée de sa conjointe.

Plusieurs femmes vont, par ailleurs, lier la transmission du nom du père à la reconnaissance de son rôle, évoquant la grossesse et l'accouchement comme constituant déjà une mise en évidence de leur propre statut de mère. 
Elisa Robichaud justifie ainsi le choix du nom du père pour ses enfants et considère que la transmission de son nom n'était pas pour elle un enjeu féministe. Elle dit :

Je savais que j'aurais pu donner juste le mien, ou juste celui de Jean-Louis, ou les deux noms ensemble, puis dans l'ordre qu'on voulait. Mais c'était pas une question. Je sais que c'est mes enfants, je les ai portés. Je n'ai pas besoin de mon nom de famille. (Elisa Robichaud, 35 ans, conjointe de fait, un fils et une fille, décoratrice, Montréal)

Elle ajoute :

Les mères, on prend tellement d'importance dans l'éducation des enfants, que le nom, non, vraiment, moi, ça, je laisse aller, puis je ne me sens pas mal du tout. Ça fait que je ne vois pas ça comme une pensée féministe. J'avoue que c'est vieux jeu, mais c'est même pas une question. C'est comme ça que ça marche.

On trouve des propos analogues chez Lisa Boucher-Rioux, héritière d'un nom double et qui parle de la difficulté de porter deux noms. Très jeune, elle a opté pour l'usage exclusif du nom de son père. Bien qu'elle ne soit pas opposée à l'idée de transmettre les noms des deux parents, à condition, comme elle le dit, «qu'on s'en tienne qu'à une seule appellation [nomination] pour éviter la confusion ", elle a choisi de donner le nom de leur père à ses enfants. Elle raconte :

Ça reste que c'est mes enfants. [...] qu'ils portent n'importe quel nom, ça va rester mes enfants. Je (ne) vois pas l'importance, à part le côté de l'ego. Comme, Boucher-Rioux, il y a juste moi et mon frère qui ont ce nom-là. [...] C'est sûr que si mon [frère] a pas d'enfant, ça va finir là. Mais à quel point c'est... [important] je le sais pas. " (Lisa Boucher-Rioux, 27 ans, mariée, au foyer, trois filles, vit en banlieue de Montréal)

Cette reconnaissance par les mères du rôle paternel en permettant que soit donné à leur enfant uniquement le nom de famille du père s'affirme davantage encore quand ce père a été lui-même abandonné ou négligé par son père.

Jeanne Langevin (29 ans, conjointe de fait, employée, une fille, vit en région), opposée à l'attribution d'un nom double, aurait aimé transmettre son nom à ses filles en alternant le nom de son conjoint et le sien à chacun de ses enfants. Mais son conjoint John Mac Donald, ayant porté le nom de 
sa mère et en ayant souffert à cause de l'absence de son père et parce qu'il ne portait pas le même nom que ses frères, tient fermement à transmettre son nom à tous ses enfants. Pour cette raison, elle s'est ralliée à la décision de s'en tenir à la transmission patronymique.

André Dusseault aurait, au contraire, voulu abandonner son nom pour celui de sa conjointe, expliquant :

J'ai eu pendant le processus de grossesse ce questionnement-là à savoir : est-ce que je propage ce nom-là? Est-ce que je vais être fier que ma fille porte ce nom-là, avec le passé auquel est rattaché ce nom-là, le passé peu glorieux de mon père et peu glorieux de sa famille? (André Dusseault, 37 ans, conjoint de fait, infographiste, une fille, vit à Montréal).

Mais sa conjointe tient à ce qu'il donne son nom à son enfant pour faire reconnaître son statut de père, et aussi d'une certaine manière la stabilité de leur couple.

\section{Un patronyme, par refus de choisir entre les parents ou les lignées}

Parmi les parents qui ont choisi de donner à leur enfant le seul nom de son père, on trouve deux femmes qui portent un nom composé. Elles adhèrent à cet héritage féministe reçu de leurs parents. Leurs conjoints respectifs veulent transmettre leur propre nom, mais seraient d'accord pour que leur conjointe transmette aussi le sien. Elles choisissent toutefois de ne pas le faire.

Pour Geneviève Gagnon-Larouche, son nom est associé au conflit familial qui a empoisonné son enfance. Ses parents se sont séparés quelques mois après sa naissance. Par la suite, chaque lignée lui a donné un nom différent, comme pour nier symboliquement l'existence de l'autre. Pour se protéger de ces atteintes à sa propre identité, elle a dû développer une habileté à jongler avec les composantes de son double nom. Aujourd'hui, les deux parentèles s'entendent mieux et elle est très liée avec les deux clans. Enceinte, elle voit poindre toutefois une résurgence de cette querelle du nom et décide sans hésiter d'appeler son enfant du nom de son père. Elle explique :

Mais moi j'ai coupé ça. J'ai dit, « regarde, [...] on va être heureux parce que justement on va créer notre famille à nous autres. Ça fait que je trouvais ça bien. C'était moins compliqué. Un moment donné ça suffit, là. (Geneviève Gagnon-Larouche, 33 ans, laborantine, un fils, vit à Montréal). 
C'est plutôt au niveau des seconds prénoms qu'elle s'amuse à recréer et croiser les liens intergénérationnels, remontant même à son arrière-grandpère.

Aurélie Thériault-Lizée avait, elle aussi, deux parents qui partageaient des idées féministes et qui lui ont transmis leurs deux noms :

Mon père était complètement pour ça. Mon père, c'était un homme féministe. Bien, ce l'est encore. Il fait partie des hommes féministes, là. Je pense qu'il était enchanté par l'idée de se battre pour ça, pour cet idéal-là. Oui, oui, c'était clair que c'était important pour les deux. (Aurélie Thériault-Lizée, 33 ans, mariée, enseignante, une fille, vit à Montréal).

Elle et ses sœurs étaient fières de porter leurs noms et elle pensait bien donner à son enfant des noms doubles. Une fois enceinte, comme son conjoint veut également transmettre son nom, elle s'avoue incapable de choisir quelle partie de son double nom elle voudrait transmettre. L'enfant a donc reçu le nom de son père. Ainsi, elle souligne toute la difficulté pour la seconde génération née après la réforme de notre système de nomination d'assumer l'impossible transmission des noms qui arrivent en surplus :

Si j'avais été avec un gars qui a deux noms de famille aussi [...] On n'aurait pas eu le choix de choisir, là, parce que c'est sûr qu'il aurait pas eu le nom composé de mon chum, là. Je veux dire, il y a des limites (rires). C'est comme si c'était une bonne idée théorique, le double nom, magnifique pour le principe [...] Mais un $[\ldots]$ casse-tête quand tu te retrouves après ça à (ne) pas pouvoir en passer un des deux parce qu'on peut pas avoir les quatre, ou les trois : il aurait pas pu s'appeler Thériault-Lizée-Doiron.

Lorsque les femmes n'arrivent pas à choisir la partie de leur nom double à transmettre, elles semblent ainsi solutionner leur dilemme en choisissant de transmettre à leur enfant le nom de son père.

\section{Transmettre un nom double}

Parmi les cinq couples ayant donné un nom double à leur enfant, on observe trois types de motivation : soit inscrire l'enfant dans une lignée tout en reconnaissant l'autre parent, soit par reconnaissance des deux lignées et la recherche d'un nom unique, soit suivant le principe d'égalité des parents. 


\section{Pour inscrire l'enfant dans une lignée tout en reconnaissant l'autre parent}

La transmission d'un nom double ne représente pas nécessairement pour les parents une rupture avec la tradition. Elle peut en effet s'opérer sans modifier fondamentalement la logique de transmission d'un seul nom qui va perdurer, perpétuer la lignée, tout en accordant une reconnaissance secondaire à la place de l'autre parent dans la formation de la famille. Cette situation est mise en évidence dans l'analyse de deux entrevues.

Tout d'abord dans celle réalisée avec Alberto Mora Gonzales, originaire du Nicaragua, marié à une Québécoise. Dans son pays et sa culture, la transmission du nom des deux parents est la règle, bien que la préséance soit accordée au patronyme à la génération suivante. Concernant le nom de sa conjointe, il explique :

Je pense que Carla est heureuse aussi d'avoir son nom dans le nom légal de nos enfants.

Par contre, il espère aussi que son fils aîné transmettra son nom, parce que :

C'est comme la seule chose qui va rester après. C'est comme un héritage. [...] Mon fils, j'espère qu'il va le donner à mon petit-fils et après, ça va continuer comme ça. (Alberto Mora Gonzales, 30 ans, marié, journalier, un fils et une fille, vit à Montréal).

Dans la seconde entrevue faisant état de ce type de transmission, l'homme rencontré, Joël Fortin ( 35 ans, conjoint de fait, menuisier, quatre filles et un garçon, vit en région), dit n'avoir pas souhaité transmettre son nom parce qu'il n' "aime pas ce qui s'y rattache». Sa conjointe, fille unique, voulait au contraire transmettre le sien car « elle voulait que son nom se perpétue ». Par contre, comme il le dit, «ma conjointe trouvait ça important que mes enfants aient une partie de moi dans leur nom ». Sa conjointe l'a persuadé ainsi de mettre son nom en deuxième, avançant que leurs enfants laisseraient tomber l'usage de ce deuxième nom dans la vie courante.

\section{Par reconnaissance des deux lignées et la recherche d'un nom unique}

Catherine De La Chênelière-Flynn met rapidement en avant au moment de l'entrevue la rareté de son nom et l'unicité qu'il lui donne. Elle dit "Moi, mon nom, je suis la seule qui le porte au monde. Alors si quelqu'un parle de Catherine De La Chênelière-Flynn, c'est sûr qu'il parle de moi » 
(Catherine De La Chênelière-Flynn, 32 ans, conjointe de fait, paysagiste, un fils, vit à Montréal)

Si le choix des prénoms de leur enfant a fait l'objet d'échanges faciles avec son conjoint dans un contexte où le couple rejette les prénoms trop originaux, c'est surtout le choix du nom de famille qui s'est avéré un processus complexe. Dans un premier temps, ils en sont arrivés à l'idée de transmettre le nom de la mère à une fille et le nom du père à un garçon. Elle voulait de son côté transmettre le nom de sa mère, pour «la lignée maternelle » mais aussi pour la rareté de son nom car comme elle le dit: « des De la Chênelière, il (n) 'y en a pas des tonnes non plus ».

Au milieu de sa grossesse, elle réalise que si elle a une fille et un garçon, ils ne porteront pas le même nom. Elle décide alors de militer ardemment pour la transmission d'un double nom, «en pointant l'unicité » du nom de leur enfant : "Adrien De la Chênelière-Descôteaux, c'est sûr qu'il y en a pas deux nulle part».

Ce projet de transmission d'un double nom poursuivi avec ténacité ne va pas sans objections de la part du conjoint et de l'entourage. Le point de vue de son conjoint apparaît en filigrane dans son discours et révèle qu'il n'avait jamais pensé à cette question avant, qu'il ne considère pas les noms doubles comme de vrais noms et qu'il se préoccupe des sentiments de son propre père. Autour d'eux, certains lui disent, comme l'a fait son conjoint au moment de signer le formulaire de déclaration de naissance: "Es-tu bien sûre? Tu ne trouves pas que c'est trop long?" Si la perspective de créer une famille dont les enfants ne porteraient pas le même nom a ébranlé son projet initial de survie des lignées paternelles par les garçons et maternelles par les filles, l'évocation de sa demi-sœur qui ne porte pas le même nom qu'elle, lui sert aussi à formuler l'intérêt d'un nom double pour la mère et l'enfant advenant la séparation du couple. Pour l'ordre des noms, Catherine affirme qu'elle n'avait pas de préférence mais que c'est son conjoint qui a choisi parce qu'il « trouvait que ça sonnait mieux De La Chênelière-Descoteaux que Descoteaux- De La Chênelière ».

\section{Suivant le principe d'égalité des parents}

Le troisième type de motivation repéré pour la transmission d'un nom double est de souscrire ainsi au principe d'égalité des parents. Ce choix est mis en évidence à travers l'analyse de deux entrevues.

On retrouve tout d'abord ce type de motivation chez Emilie DoréMountain. Cette jeune femme a reçu un nom composé à la naissance, mais elle n'a pas connu son père et elle n'utilise pas son nom au quotidien. Elle se présente toujours comme Émilie Doré. 
Pour cette femme, le nom trace l'histoire des parents de l'enfant. Elle dit :

Dans mon cas, mon nom de famille est très porteur de ce que je suis, d'où je viens, de mon héritage, puis de ce que je n'ai pas aussi. Mon nom représente bien ce qu'il [n'] y a pas chez moi, c'est-àdire un père; aussi, je [ne] porte pas [au quotidien] le nom de mon père. (Emilie Doré-Mountain, 39 ans, conjointe de fait, architecte, une fille, vit à Montréal).

Elle veut ainsi que sa fille ait trace de ses deux lignées et histoires parentales, qu'elle n'ait pas trace d'une absence, contrairement à elle. Expliquant l'ordre des noms donnés à son enfant, elle dit: «On a essayé l'un, on a essayé l'autre, puis musicalement ça sonnait mieux Charbonneau-Doré, alors c'est ce qu'on a choisi...»

L'entretien réalisé avec Marc Tessier-Morton (32 ans, conjoint de fait, médecin, un fils, vit à Montréal) met aussi en évidence une recherche d'équité entre les conjoints dans le nom transmis à leur enfant. Parlant de son couple, il dit d'ailleurs: «l'équité est un mode de pensée qui est omniprésent dans notre relation ». Sa conjointe souhaitant transmettre son nom, Marc Tessier-Morton doit alors choisir de ne transmettre qu'une partie de son nom composé, soit Tessier. Ce choix ne s'est pas fait au nom de la norme culturelle, ni de sentiments envers ses parents, mais au nom de la sonorité et d'un soupçon de nationalisme, car c'est de son père qu'il tient la partie anglaise de son nom (Morton). Il regrette tout de même d'avoir sacrifié une trace du passé familial, de «ne pas avoir gardé une trace de ce nom quelque part ", notamment dans les prénoms secondaires de son enfant. L'ordre des noms transmis à son fils semble aussi avoir été choisi sur la sonorité. Parce qu'il regrette un peu d'avoir laissé tomber le nom de son père, il songe un moment à inclure Morton dans le nom de famille d'un autre enfant. Sa conjointe s'y oppose toutefois appelant la primauté du principe d'un nom de famille identique pour tous les enfants.

Tout comme Marc Tessier-Morton, Catherine De La ChênelièreFlynn avait émis l'idée de la transmission du nom par alternance, mais l'hétérogénéité des noms dans la fratrie que ce choix aurait entraînée leur a fait changer d'avis.

\section{Transmettre un nom différent à la fratrie}

Le système québécois de nomination n'oblige pas les couples à choisir un même nom de famille pour tous leurs enfants communs, ce qui le rend 
fortement individualisant. Les parents peuvent donner un nom composé à l'un des enfants et pas à l'autre, modifier d'un enfant à l'autre l'ordre des noms qui forment le nom composé, ou encore donner le nom du père à l'un et celui de la mère à l'autre, selon un modèle d'alternance dont nous avons rencontré deux cas de figure : le nom transmis dépend du sexe de l'enfant et les père et mère transmettent leur nom à tour de rôle. Pour les parents rencontrés qui ont choisi un tel modèle d'alternance, il s'accompagnait d'un rejet du double nom considéré comme trop compliqué.

\section{Le nom transmis lié au sexe de l'enfant}

Des parents ont choisi de donner à un garçon le nom de son père et à une fille, celui de sa mère (création de lignées patronymique et matronymique).

C'est selon ce modèle d'alternance que le fils de Liliane Caron-Jolin (31 ans, anthropologue, séparée, un fils, Montréal) a reçu par exemple, le nom de son père. Le couple n'a toutefois pas eu d'autres enfants, car il s'est séparé peu après la naissance de leur enfant. S'ils avaient eu une fille, il était prévu que Liliane lui transmette seulement le segment de son propre nom composé qui lui vient de sa mère : Caron. Elle aurait ainsi, dit-elle, créé « une lignée de filles». Cette transmission lui aurait aussi permis du même coup, comme elle l'explique, de laisser tomber le nom de son père, qui a été fort peu présent dans sa vie.

\section{Les père et mère transmettent leur nom à tour de rôle}

Le second cas de figure est celui où chaque parent, à tour de rôle, nomme un enfant, en commençant soit par le parent du même sexe que l'aîné, soit par tirage au sort.

\section{Le nom de l'aîné est donné par le parent de même sexe}

Le premier enfant de Marie-Lyne Dominique et Daniel Bordeleau étant un garçon, le couple a décidé de lui transmettre le nom de son père. La mère lui a donné son nom, qui est aussi un prénom, comme second prénom. À ce sujet, elle dit : «ça va faire vraiment une partie de moi, un petit peu de son identité » (Marie-Lyne Dominique, 35 ans, conjointe de fait, un fils et une fille, informaticienne, Montréal).

Leur second enfant est une fille, qui porte comme convenu le nom de sa mère. Pour qu'il y ait aussi «quelque chose de la famille de son conjoint ", «sa famille à lui », l'enfant a reçu comme second prénom celui 
de la grand-mère maternelle de son père. Une symétrie dans la transmission des noms et prénoms secondaires des lignées s'observe ainsi.

\section{Nom transmis par alternance après tirage au sort du nom de l'aîné}

Julie Paquette et McLeod Laurens ont quant à eux tiré au sort pour décider lequel d'entre eux transmettrait le premier son nom. C'est elle qui a gagné et le hasard a fait qu'ils ont eu d'abord une fille nommée Paquette, puis un garçon nommé Laurens. Julie n'a pas l'ambition de perpétuer ainsi son nom. Elle ne s'attend pas du tout à ce que sa fille le retransmette un jour à sa petite-fille affirmant : "ça ne nous appartient plus après »(Julie Paquette, 32 ans, mariée, cinéaste, une fille et un fils, vit à Montréal).

Par ailleurs, elle insiste pour dire que la stratégie de nomination choisie par le couple ne se fonde pas sur une association stéréotypée mèrefille, père-fils. Au contraire, dit-elle, le troisième enfant du couple s'appellera probablement Paquette, même si c'est un garçon, pour bien en faire la démonstration à ceux qui en douteraient.

Comme précédemment, l'établissement d'une symétrie inversée dans le choix des prénoms est adopté. Ainsi, les prénoms secondaires de la fille de Julie Paquette sont le nom de famille de son père (Laurens) et le prénom de sa grand-mère paternelle. Inversement, l'enfant qui porte le nom de famille de son père a reçu comme prénoms secondaires celui de sa grandmère maternelle (au masculin) et une syllabe d'un des prénoms de sa mère.

L'identité familiale commune se marque ainsi à travers les noms et prénoms des enfants considérés dans leur ensemble. Cependant, elle passe inaperçue aux yeux des observateurs extérieurs : ces références identitaires sont de l'ordre du privé, sinon de l'intime.

\section{CONCLUSION}

Si les statistiques récentes montrent une hausse de l'attribution du nom du père dans le processus de nomination, les entretiens soulignent pour leur part l'importance accrue de la fonction connotative du nom dans les pratiques actuelles de nomination (Gutman, 2000).

En obligeant les parents à choisir le nom qu'ils veulent donner à leur enfant, la réforme du Code civil de 1980 a contribué à affaiblir l'efficacité de la fonction dénotative du nom en tant que marqueur de filiation et l'importance qui lui est accordée. Ainsi, choisir d'attribuer un nom non conventionnel - un nom double ou un nom que les autres enfants de la fratrie ne partagent pas - peut d'abord répondre au souci d'appliquer un 
principe d'égalité entre les parents et d'affirmer la reconnaissance des lignées paternelles et maternelles. Il peut aussi dans le cas du modèle de transmission alternée s'appuyer sur un principe de différenciation des sexes (lignées patronymiques et matronymiques) ou se fonder sur un principe d'égalité individuelle en postulant l'indifférence des sexes (tirage au sort). Les motivations associées à la transmission du nom du père sont également variées, et ne se limitent pas aux discours dénotant une inégalité entre les sexes dans l'appropriation symbolique des enfants, ni un retour vers un conservatisme patrilinéaire. Transmettre un patronyme peut être voulu en référence à des normes du milieu d'appartenance, de reconnaissance d'une filiation paternelle ou d'une identité paternelle exprimée à travers le patronyme mis en balance avec la grossesse et l'accouchement pour la filiation maternelle (Théry, 2002).

On voit ainsi toute la complexité entourant la question de l'égalité ou pas des sexes dans le choix ou le refus d'un nom patronymique ou double. La réforme législative sur le nom au Québec a contribué à transformer les connotations associées au nom, celui hérité exclusivement d'une mère, par exemple, mais aussi à réfléchir à la question de l'égalité entre les sexes, où le patronyme pourrait être choisi pour compenser une asymétrie biologique.

\section{BIBLIOGRAPHIE}

Bergeron, D. 1990. «Un espace autre pour le désir », Santé mentale au Québec, 15, $1: 145-164$.

Bouchard, G., M.-A. Desjardins-Ouellette, F. Markowski et K. KoulaDJIAN. 1985. «La distribution des patronymes au Québec: témoins des dynamiques de population", Anthropologie et sociétés, 9, 3:197-218.

Brunet, G., P. Darlu et D. Barbero. 2008. «Patronymes, propriété et mobilité géographique. L'avant-pays savoyard du $\mathrm{xv}^{\mathrm{e}}{ }^{\mathrm{e}}$ au $\mathrm{xx}^{\mathrm{e}}$ siècle », Histoire et Sociétés Rurales, 30-2: 67-90.

Charton, L. et D. Lemieux. 2015. "Quand les parents choisissent noms et prénoms : pratiques et rites de nominations au Québec du $\mathrm{XxI}^{\mathrm{e}}$ siècle ", Recherches familiales, 12 : 113-124.

Chen, K. et L. Cavalli-Sforza. 1983. «Surnames in Taiwan. Interpretation Based on Geography and History », Human Biology, 55:367-374.

Conseil du Statut de la Femme (CSF). 1979. Mémoire présenté à la commission parlementaire sur la réforme du droit de la famille. Québec, CSF.

Cousteau Serdong, F. 2008. «Le Québec, paradis de la généalogie et "re-père" du patriarcat : où sont les féministes? De l'importance d'aborder la généalogie avec les outils de la réflexion féministe », Recherches féministes, 21, 1: 131-147. 
Darlu, P., A. Degioanni et J. Ruffié. 1997. «Quelques statistiques sur la distribution des patronymes en France », Population, 52, 3: 603-634.

Darlu, P. et A. Degioanni. 2007. «L'origine géographique des migrants par la méthode patronymique », L'Espace géographique, 3 : 251-265.

Darlu, P., G. Bloothooft, A. Boattini, L. Brouwer, M. Brouwer, G. Brunet, P. Chareille, J. Cheshire, R. Coates, K. Dräger, B. Desjardins, P. Hanks, P. Longley, K. Mandemakers, P. Mateos, D. Pettener, A. Useli et F. Manni. 2012. "The Family Name as Socio-Cultural Feature and Genetic Metaphor: From Concepts to Methods », Human Biology, 84-2 : 169-214.

Deleury, É. et D. Goubau. 1997. Le droit des personnes physiques (2 édition). Éditions Yvon Blais.

Desjardins, B., A. Bideau, G. Brunet, H. Charbonneau et J. Légaré. 2001. « De France en Nouvelle-France : les patronymes québécois hier et aujourd'hui », dans G. Brunet, P. Darlu et G. Zei (dir.), Le patronyme, histoire, anthropologie, société, Paris, CNRS Éditions : 203-216.

Duchesne, L. 2001. "Vers un système matrilinéaire? Le choix du nom de famille au Québec », dans G. Brunet, P. DARLu et G. ZeI (dir.), Le patronyme, histoire, anthropologie, société, Paris, CNRS Éditions : 132-151.

Duchesne, L. 2006. Les noms de famille au Québec. Aspects statistiques et distribution spatiale. Québec, Institut de la statistique du Québec.

Feschet, V. 2004. « La transmission du nom de famille en Europe occidentale (fin $\mathrm{xx}^{\mathrm{e}}$-début $\mathrm{xxI}^{\mathrm{e}}$ siècles) », L'Homme. Revue française d'anthropologie, 169: 61-88.

Fine, A. et F.-R. Ouellette (dir.). 2005. Le nom dans les sociétés occidentales contemporaines. Toulouse, Presses universitaires du Mirail.

Garneau, B. 1985. "Identité et noms de personne à Bois-Vert (Québec) », Anthropologie et Sociétés, 9-3:33-35.

Gouvernement DU QuÉBec. 2000. Loi instituant un nouveau Code civil et portant réforme du droit de la famille, LQ 1980, c. 39. Québec, Gouvernement du Québec.

Gutmann, D. 2000. Le sentiment d'identité. Étude de droit des personnes et de la famille. Paris, LGDJ.

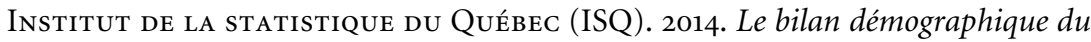
Québec. Québec, ISQ.

LAmesta, J. et M. BADDELEy. 2012. «Au nom du père et de la mère : Étude comparative sur le droit du nom dans la perspective du droit du nom 2013 ", dans Le droit civil dans le contexte international, Genève, Schulthess Éditions Romandes : 77-107.

Le Gall, J. et D. Meintel. 2005. «Pratiques de nomination dans les unions mixtes à Montréal. L'affichage des multiples appartenances de l'enfant », dans A. FinE et F.-R. Ouellette (dir.), Le nom dans les sociétés occidentales contemporaines, Toulouse, Presses universitaires du Mirail : 191-212. 
Lemieux, D. 2005. «Nommer le premier enfant. Discours et pratiques de parents québécois dans un contexte de changements familiaux et juridiques ", dans A. Fine et F.-R. Ouellette (dir.), Le nom dans les sociétés occidentales contemporaines, Toulouse, Presses universitaires du Mirail : 163-189.

Louis-PéchA, A. 2010. «Les incidences de la réforme de la filiation sur le nom de famille ", Recherches familiales, 1, 7 : 59-68.

Lucchetti, E., M. Tasso, I. Amoruso et G. Caravello. 2011. « The border effect in surname structure : an Italian-Slovenian case study », Human Biology, 83-3 : 393-404.

Mellet, J.-F. 2000. Le régime contemporain de l'attribution et du changement de nom au Québec: le "grand bond en avant " d'une institution de droit civil? McGill University, Institut de droit comparé [Thesis in partial fulfilment of the requirement of the degree of L.L.M.]

Ouellette, F.-R. 2013. "Choisir un nom de famille : un choix multiple et un véritable casse-tête! », dans M. JÉzéQuel et F.-R. Ouellette, De quoi vont hériter nos enfants ?, Montréal, Fides : 225-234.

Ouellette, F.-R. et J. Saint-Pierre. 2011. «Parenté, citoyenneté et état civil des adoptés ", Enfances, Familles, Générations, 14 : 51-76.

Roch, H. 1949. Actes et registres de l'État civil et rectification. Montréal, [s.é.].

Roy, L. 1959. De la tenue des registres de l'État civil dans la province de Québec, suivi des Règles et techniques relatives aux registres de l'État civil. Montréal, Thérien Frères limitée.

Théry, I. 2002. « Le nom, entre préséance et préférence », Esprit, 2 : 110-118.

\begin{abstract}
Laurence CHARTON, Louis DUCHESNE, Denise LEMIEUX and Françoise-Romaine OUELLETTE A return to patronymics in Quebec, 2005-2010: behind the numbers - complex discourses between equality, identity and filiation.

Since 1981, as a consequence of the recognition of the principle of equality between parents, the Civil Code in Quebec has allowed a child to be given the name of its father or that of its mother, or the two names together. A person given a composite name may pass it on to his or her child, in its entirety or in part, and combined or not with the simple or the composite name of the other parent (making 16 possible options in all). This article offers a review of this legal reform through its impact on the dynamics of name transmission, based on data collected after the new legislation (from 1980 to 2010), and on 25 interviews about the birth of their first child, carried out with mothers or fathers of children born during the period from 2008 to 2013, including parents with double names. The analysis shows, on the basis of the available figures, that an increase in double names during the 1980 s is being succeeded by a rise in last decades in attributions of the father's name alone. Although the findings cannot be generalised, evidence from interviews suggests a growth in the importance of the connotative function of discourses surrounding the naming process, in which issues of filiation, identity and equality are involved in a complex set of inter-relations.
\end{abstract}

